\title{
Buenas prácticas en la gestión del servicio a cruceros de los principales destinos del Caribe. Acciones para su mejora en el destino La Habana
}

Good practices in the management of the cruise services in the main Caribbean destinations. Actions for its improvement in the Havana destination

Claudia Pérez García. ${ }^{1}$, Yoan Hernández Flores. ${ }^{2}$, Maité Rodríguez González. ${ }^{3}$ \& Luis Efraín Velasteguí López. ${ }^{4}$

Recibido: 09-07-2020 / Revisado: 11-08-2020 / Aceptado: 11-09-2020 / Publicado: 03-10-2020

\begin{abstract}
Resumen.
DOI: https://doi.org/10.33262/concienciadigital.v3i4.1426

Dentro del crecimiento alcanzado por el turismo de cruceros, se destaca como primer destino mundial el área del Caribe. Ubicada en este contexto, se encuentra la isla de Cuba, cuyo desarrollo en el turismo de cruceros ha estado condicionado por las medidas tomadas a lo largo de los años por el gobierno de los Estados Unidos. La Habana, como principal puerto de la isla en la recepción de cruceros, presenta una deteriorada infraestructura; una escasa oferta extrahotelera; así como cifras inferiores en cuanto a llegadas de cruceristas y gastos de los mismos en el destino, en relación a destinos líderes del Caribe. Por tanto, la presente investigación tiene el objetivo de diseñar un conjunto de acciones que permitan mejorar la gestión del servicio a cruceros en el destino. Para esto, se empleó una metodología que permitió la identificación de las buenas prácticas de destinos del Caribe, líderes en la actividad, donde fueron empleados métodos teóricos y empíricos como el análisis bibliográfico, la entrevista a especialistas del sector y el criterio de expertos. De esta manera, los resultados obtenidos permitieron conocer la brecha de desempeño actual entre La Habana y destinos líderes del Caribe, así como la identificación de las buenas prácticas que estos realizan en la gestión del servicio a cruceros. Por último, se diseñaron 22 acciones que
\end{abstract}

\footnotetext{
${ }^{1}$ Facultad de Turismo, Universidad de La Habana. La Habana, Cuba. claudia_perez@estudiantes.ftur.uh.cu

${ }^{2}$ Facultad de Turismo, Universidad de La Habana. La Habana, Cuba. yoan961122@gmail.com

${ }^{3}$ Facultad de Turismo, Universidad de La Habana. La Habana, Cuba. mrodriguez@ftur.uh.cu

${ }^{4}$ Ciencia Digital Editorial, Ambato, Ecuador, luisefrainvelastegui@cienciadigital.org
} 
contemplan la implementación de estas buenas prácticas en la gestión del servicio a cruceros en La Habana.

Palabras claves: Gestión del servicio a cruceros; Caribe; La Habana; buenas prácticas; acciones.

\begin{abstract}
.
Within the growth achieved by cruise tourism, the Caribbean area stands out as the first world destination. Located in this context is the island of Cuba, whose development in cruise tourism has been conditioned by the measures taken over the years by the United States government. Havana, as the main port of the island for the reception of cruise ships, presents a deteriorated infrastructure; a scarce non-hotel offers; as well as lower figures in terms of cruise ship arrivals and expenses at the destination, in relation to leading Caribbean destinations. Therefore, this research has the objective of designing a set of actions to improve the management of the service to cruise ships in the destination. For this, a methodology was used that allowed the identification of good practices in Caribbean destinations, leaders in the activity, where theoretical and empirical methods such as bibliographic analysis, interview with specialists in the sector and expert judgment were used. In this way, the results obtained allowed to know the current performance gap between Havana and leading destinations in the Caribbean, as well as the identification of the good practices that they carry out in managing cruise service. Lastly, 22 actions were designed that contemplate the implementation of these good practices in the management of cruise service in Havana.
\end{abstract}

Keywords: Management of the service to cruise, Caribe, Havana, good practices, actions.

\title{
Introducción.
}

El turismo de cruceros ha experimentado un crecimiento significativo en las últimas décadas dentro del sector del turismo a nivel mundial, convirtiéndose en una de las áreas con mayor proyección en volumen de pasajeros e ingresos.

Esta actividad continúa teniendo un impacto positivo en las comunidades de todo el mundo al mantener aproximadamente 1177000 empleos, equivalentes a 50.4 mil millones de dólares en sueldos y salarios y 150 mil millones de producción total en todo el mundo en 2018, Asociación Internacional de Líneas de Cruceros, (CLIA, 2019).

El Caribe constituye el primero de los mercados receptores del turismo de crucero, principalmente por el atractivo que representa su clima cálido y soleado, sus espectaculares playas y sus interesantes excursiones. Esta posición como destino de cruceros se debe fundamentalmente a su cercanía respecto al mercado emisor de cruceristas más importante, los Estados Unidos (Ruiz y Parada, 2017). 
En este contexto deben mencionarse destinos como Cozumel, Bahamas, Jamaica, Puerto Rico y Gran Caimán que constituyen, según las estimaciones más recientes realizadas por el Business Research and Economic Advisors (BREA) para la Florida Caribbean Cruise Association (FCCA) en el 2019, puntos de referencia de esta gran región, pues el número de pasajeros de cruceros es determinante.

Cuba también ha estado inmersa desde 1993, en la práctica del turismo de cruceros, el cual ha sido un complemento importante del turismo de estancia. En el 2018, recibió más de 800000 pasajeros que visitaron la isla en cruceros, lo cual representó un aumento del 29,2\% con respecto al 2017 (Chevalier, 2019).

Según el sitio web Excelencias Cuba (2018), durante el primer semestre los buques Cruceros han efectuado 178 escalas en el Puerto de La Habana, varios de estos buques incluyen en sus itinerarios visitas a los puertos de Cienfuegos y Santiago de Cuba; todos los itinerarios de cruceros a Cuba consideran a La Habana, una visita obligada. Entre las compañías de Cruceros que incluyeron a Cuba en sus itinerarios, cuatro de ellas con base en Florida, han realizado 84 escalas. Otros armadores han realizado 94 escalas en el puerto de La Habana, además de incluir en sus itinerarios varios puntos de visita en los litorales costeros cubanos.

Las diferentes compañías se han visto atraídas por la privilegiada situación geográfica del archipiélago, así como por su cultura, su pueblo, su patrimonio histórico cultural y su seguridad; que constituyen en su conjunto ventajas competitivas. Sin embargo, presenta cifras inferiores con relación a volumen de pasajeros y gasto de los mismos en el destino en comparación con los destinos del Caribe mencionados anteriormente.

A pesar de ser La Habana el puerto principal de la isla, la gestión de servicio a cruceros en la misma se ha visto afectada por diferentes factores en períodos posteriores. Cabe señalar, entre ellos, la deteriorada e insuficiente infraestructura que no permite recibir grandes volúmenes de buques y clientes. Además, la inadecuada relación entre las agencias de viaje y los prestatarios de servicios, que ha dado paso a problemas en la calidad (Rodríguez, 2019).

Una de las principales causas de la disminución de los cruceristas ha sido la política agresiva del gobierno de los Estados Unidos (EE.UU.) que prohíbe a los estadounidenses viajar a la isla como turistas y, a lo cual se le suma las últimas medidas que prohíben los viajes bajo la modalidad pueblo a pueblo, así como las visitas a Cuba a través de embarcaciones de pasajeros y embarcaciones recreativas, incluidos cruceros y yates, así como aviones privados y corporativos. Estas medidas repercutieron de tal manera que, en el 2019, disminuyó en un 49 \% la llegada de cruceristas a La Habana y en más de un 50 \% a Cuba, con respecto al 2018, Ministerio de Turismo, (MINTUR, 2019).

Aunque el turismo de cruceros sea, en estos momentos, una actividad difícil de desarrollar para Cuba, se deben sentar las bases de una buena gestión para el futuro; reforzando los servicios involucrados en esta actividad, y no solo para oportunidades futuras, sino también para la mejora del servicio a los cruceros que actualmente atracan en La Habana. 
Por ello, se hace necesario conocer como gestionan otros destinos el servicio a cruceros y las buenas prácticas que estos desarrollan en esta actividad. Una visión estratégica es siempre necesaria en la construcción del futuro. Además, el estudio de estas buenas prácticas se hace necesario en la dinámica del mundo actual globalizado, que genera cambios constantes e innovadores que deben ser incorporados, para de esta manera alcanzar y sostener los niveles de éxito que se esperan.

Se define entonces como objetivo general: Diseñar acciones para la implementación de las buenas prácticas de los principales destinos del Caribe en la gestión del servicio a cruceros en La Habana.

El estudio de la gestión del servicio a cruceros reviste gran importancia dado a las escasas publicaciones con referencia a este tema. Asimismo, el conocimiento de la actividad en otros destinos y sus formas de gestión, permiten la incorporación de técnicas novedosas, así como un posicionamiento en una industria altamente competitiva.

\section{Metodología.}

En función del ordenamiento metodológico de la presente investigación, fueron consultadas varias metodologías de benchmarking, enfocadas a la identificación de las buenas prácticas en destinos turísticos o actividades propiamente del turismo, entre ellas Camp (1993) citado en Hernández y Cano (2017), Instituto Tecnológico de Monterrey (2000), López (2010) y Developing Natural Activities (2013).

Luego del análisis de estas metodologías, se diseñó un procedimiento metodológico (Tabla 1), adaptando la propuesta de Camp (1993) citado en Hernández y Cano (2017), dado a que es una de las principales y más utilizadas, además es clara y precisa. Esta propuesta, es la única de las consultadas que posee dentro de sus fases un paso para definir qué variables o indicadores utilizar, paso necesario en la investigación al no orientarse ninguna de las metodologías consultadas a la gestión del servicio a cruceros; mientras que las demás, como se explicó anteriormente, los tienen preestablecidos

Tabla 1: Procedimiento metodológico para el desarrollo de la investigación

\begin{tabular}{|c|c|c|}
\hline Fases & Etapas & $\begin{array}{l}\text { Métodos, técnicas } \\
\text { y herramientas }\end{array}$ \\
\hline \multirow{5}{*}{$\begin{array}{l}\text { Fase 1: } \\
\text { Planificación }\end{array}$} & & $\begin{array}{c}\text { Revisión } \\
\text { documental }\end{array}$ \\
\hline & Definición de las variables & Consulta a expertos \\
\hline & & Coeficiente de \\
\hline & & Alpha de Cronbach \\
\hline & $\begin{array}{c}\text { Comparar los resultados internos con la } \\
\text { competencia }\end{array}$ & $\begin{array}{c}\text { Revisión } \\
\text { documental } \\
\text { Cuestionarios }\end{array}$ \\
\hline
\end{tabular}


ISSN: 2600-5859

Vol. 3, $\mathrm{N}^{\circ} 4$, p. 71-89, octubre-diciembre, 2020

Determinar problemas existentes en la gestión

del servicio a cruceros

Fase 2:

Análisis

Fase 3:

Integración
Identificar las prácticas de los destinos líderes

Determinar la brecha de desempeño de actividad
Establecer acciones para implementar las buenas prácticas identificadas
Entrevistas semi-

estandarizadas

Revisión

bibliográfica

Cuestionarios

Método Delphi

Tormenta de Ideas

Fuente: Elaboración propia a partir de la propuesta de Camp (1933) citado en Hernández y Cano (2017).

La metodología de Camp (1993) citada en Hernández y Cano (2017), se enfoca en la empresa, pero, en la presente investigación, se abordó desde la perspectiva del destino, al ser el contexto en el cual se desarrolla el problema de investigación.

En primer lugar, se definió qué proceso de producción o servicio se somete al estudio del benchmarking que en este caso es la gestión de servicios a cruceros en los destinos.

Como parte de la primera fase, se definieron las variables de estudio; para lo cual se sometieron al criterio de diferentes expertos. Los criterios de selección de los expertos para este estudio fueron el nivel de conocimiento, experiencia y estudios realizados, que determinan el cálculo de un coeficiente de competencia para la selección final.

En la investigación, se aplicó el coeficiente Alpha de Cronbach para validar la confiabilidad y validez de las variables identificadas, utilizando para ello el software SPPS Statistic versión 20.

Luego de definidas las variables por los expertos, se realizó una comparación entre los resultados internos en la gestión del servicio a cruceros en La Habana, tomando como referencia los destinos con mejores resultados en el Caribe. Esta comparación se realizó atendiendo a las variables: cantidad de llegadas de cruceristas al destino, gasto promedio y total del crucerista en el destino y porciento de satisfacción del crucerista, las cuales brindan un acercamiento al estado de la gestión del servicio a cruceros en un destino.

En segundo lugar, se identificaron aquellos destinos referentes de estudio. Para ello se tuvo en cuenta una serie de requisitos:

- Contener información útil.

- Que sea una información que esté al alcance del autor.

- Que el modo de obtener esa información sea aceptable.

- Arribo de más de un millón de cruceristas

- Prestigio internacional como destino receptor de cruceros. 
Para la recopilación de la información, se utilizaron además cuestionarios a especialistas de los destinos seleccionados sobre los aspectos más relevantes para conocer mejor a los destinos de referencia y así mejorar procedimientos.

En la segunda fase, el primer paso fue determinar los principales problemas existentes en la gestión del servicio a cruceros en La Habana, para lo cual se desarrollaron entrevistas semi-estandarizadas a expertos en la gestión del servicio a cruceros del destino. Posteriormente, con información obtenida en la etapa anterior, y nuevos cuestionarios aplicados, se identificaron que prácticas realizan los destinos líderes que puedan beneficiar al destino.

Por último, en esta fase se determinó la brecha de desempeño en la actividad, de La Habana con relación a los líderes. Para ello, los expertos determinaron la brecha existente entre los destinos por variables:

1: una brecha negativa con relación al destino líder

2: operaciones en paridad

3: una brecha positiva con relación al destino líder

Los resultados pueden determinar que se tiene una brecha negativa, positiva o con operaciones en paridad; la primera de ella significa que las prácticas de los líderes son mejores que las que desarrolla el destino; ahora bien, si se obtiene que los procedimientos del destino son mejores, entonces se debe realizar un estudio más amplio e interno, y la tercera supone que se tienen procedimientos muy similares y que las diferencias no son significativas.

Durante la tercera fase, mediante la Tormenta de Ideas, los autores, establecieron acciones donde prevalecía la iniciativa de cambio con las mejores prácticas identificadas. El principal objetivo es, transformar dichas prácticas en principios de operación, es decir, en acciones que ocasionen un cierre de la brecha que existe entre los destinos.

\section{Resultados}

\section{Fase 1: Fase de planificación.}

\section{Definición de variables.}

Una vez aplicada, procesadas las encuestas a los especialistas y determinado el coeficiente de Cronbach (Tabla 2), quedaron definidas 10 variables para el estudio a realizar.

Tabla 2: Variables definidas

\begin{tabular}{lc}
\hline \multicolumn{1}{c}{ Variables definidas } & Alpha de Cronbach \\
\hline Cantidad de arribos de cruceristas al destino & 0.880495 \\
Gasto promedio del crucerista en el destino & 0.880495 \\
Generación de empleo & 0.919444 \\
Contribución económica al destino & 0.919444 \\
\hline
\end{tabular}




\begin{tabular}{ll}
\hline Infraestructura portuaria & 0.919444 \\
Infraestructura turística & 0.919444 \\
Cantidad de servicios turísticos & 0.919444 \\
Calidad de servicios turísticos & 0.919444 \\
Rol de las agencias de viajes en la actividad & 0.919444 \\
Acceso a la tecnología & 0.919444 \\
\hline
\end{tabular}

Fuente: Elaboración propia, a partir del software SSPP Statistic versión 20.

\section{Comparación de los resultados internos en la gestión del servicio a cruceros en La Habana con los destinos líderes del Caribe}

La Habana como destino de cruceros se inserta en una de las zonas geográficas de mayor dinamismo en esta actividad, el Caribe. Su posición geográfica, constituye una ventaja competitiva, así como un atractivo, al ser uno de los pocos puertos de cruceros en los cuales al llegar los cruceristas contemplan la ciudad y todo el centro histórico sin tener que trasladarse. Junto a esto, el recibimiento del pueblo, el patrimonio, la historia y su cultura en general, la hacen un destino con grandes previsiones de desarrollo en este sector. Sin embargo, al comparar los resultados internos con los destinos líderes del Caribe, se evidencian diferencias, en donde La Habana, presenta cifras inferiores con relación a variables significativas.

Entre los principales destinos líderes de turismo de cruceros en el Caribe en el año 2019, según CLIA (2019), se encuentran: Cozumel, Bahamas, Jamaica, Gran Caimán, Puerto Rico, Costa Maya e Islas Vírgenes.

\section{Llegadas de cruceristas al destino.}

Con respecto a esta variable se encuentra en primer lugar con más de 3 millones de llegadas de cruceristas, la isla de Cozumel; seguido de las Bahamas con más de 2 millones; Jamaica, Gran Caimán, Puerto Rico y Costa Maya con más de un millón y en últimos lugares las Islas Vírgenes y La Habana con menos de un millón (Figura 1).

Figura 1: Llegadas de cruceristas a los principales destinos del Caribe. Mayo 2017- abril 2018

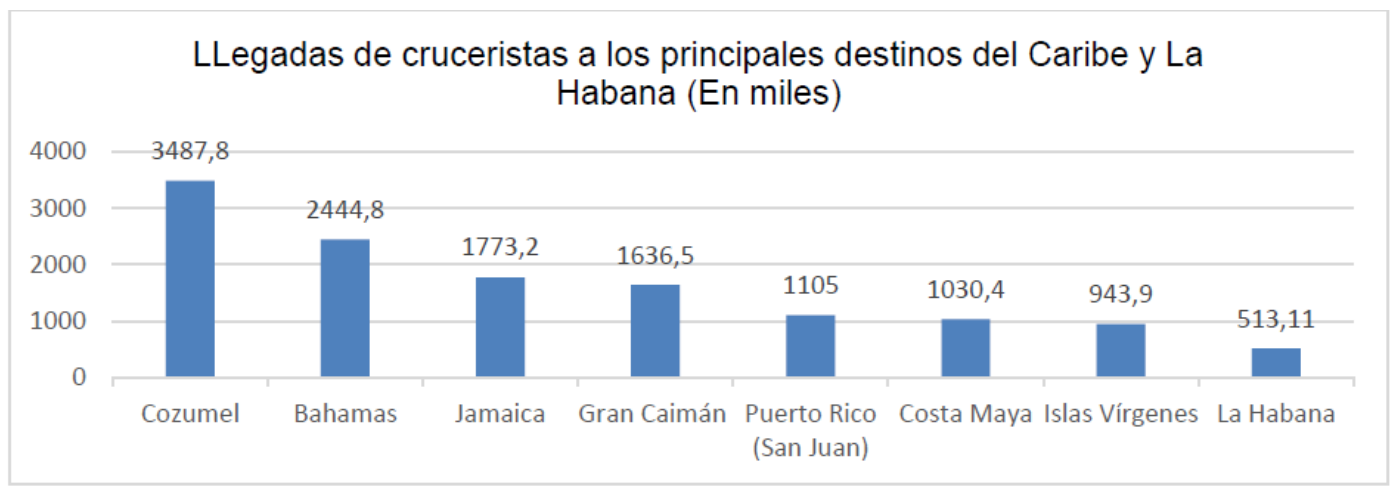


Fuente: Elaboración propia a partir de los datos de Business Research and Economic Advisors (BREA, 2018) y MINTUR (2019).

\section{Gasto promedio por crucerista y gastos totales.}

Las Islas Vírgenes son el destino con mayor gasto promedio del crucerista en el Caribe, con una cifra de 165,42 dólares, seguido de las Bahamas con \$131,95 (Figura 2). Sin embargo, el destino con más gastos totales es Cozumel con $\$ 377$ millones de dólares. Dentro de estos destinos Cuba, presenta las cifras más bajas con un gasto promedio de \$ 52 y gastos totales de \$24 millones.

Figura 2: Gasto promedio del crucerista y gastos totales. Período: mayo 2017 - abril 2018

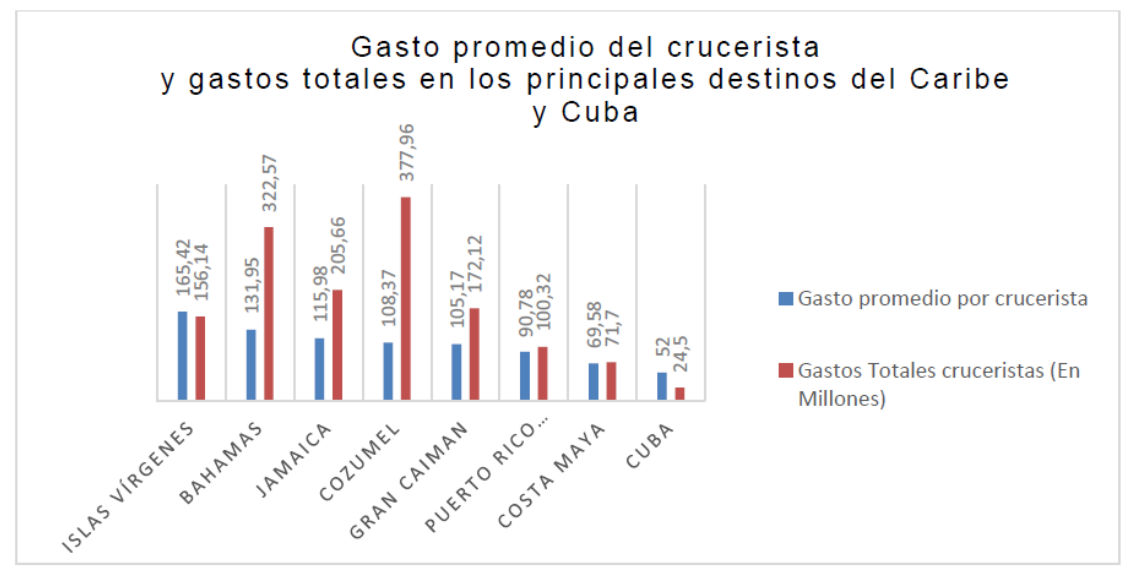

Fuente: Elaboración propia a partir de los datos de (BREA, 2018) y MINTUR (2019).

\section{Satisfacción del crucerista.}

Con respecto al por ciento de satisfacción del crucerista, La Habana presenta resultados favorables con uno de los valores más alto como se muestra la Figura 3, superando a los destinos restantes e igualando a Cozumel. Este último destino, al igual que en las otras variables, ocupa una posición destacada.

Figura 3: Por ciento de satisfacción del crucerista. Período: mayo 2017 - abril 2018.

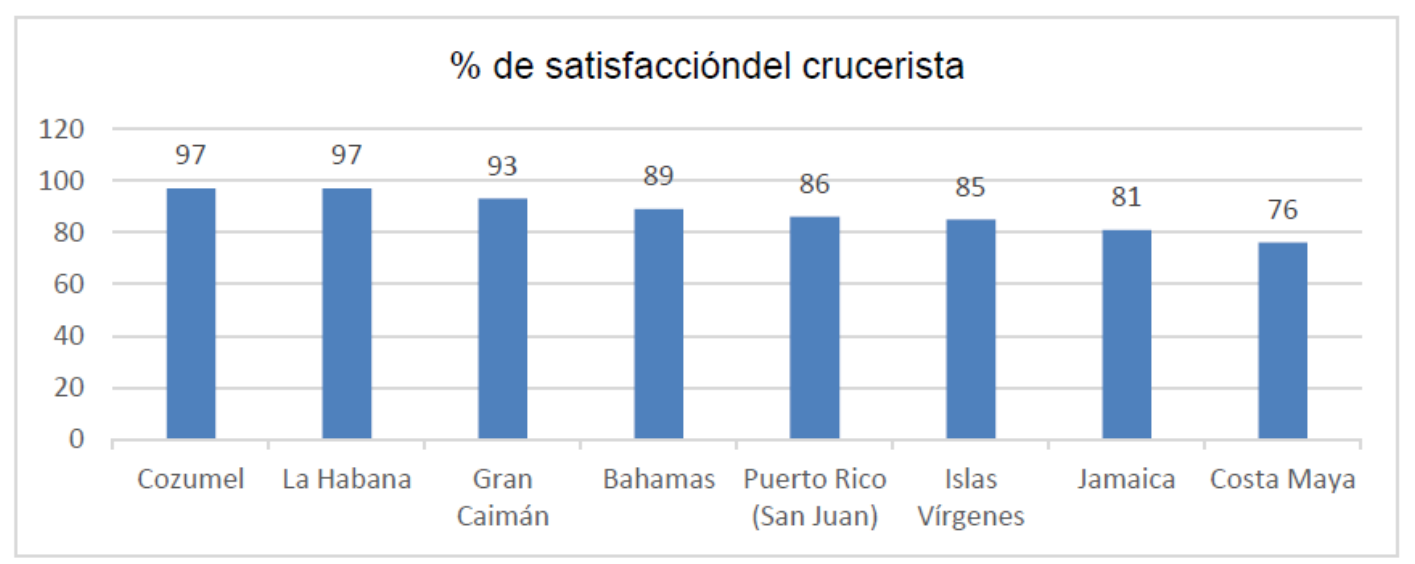


Fuente: Elaboración propia a partir de los datos de (BREA, 2018) y MINTUR (2019).

A partir de los resultados anteriores, para el desarrollo de las etapas posteriores de la investigación se seleccionaron los destinos Cozumel y Las Bahamas como mayor competencia del destino Cuba.

Estos dos destinos poseen un gran prestigio internacional, al estar entre los más populares para los cruceristas, han recibido premios y reconocimientos que lo confirman y sobrepasan la llegada de más de un millón de cruceristas anuales. Además, se incluye en itinerarios destacados de importantes compañías de cruceros que navegan el Caribe.

Al ser Cozumel un destino con un claro éxito en el sector, existen numerosas investigaciones que han llevado a cabo el estudio del desarrollo de dicha actividad. Estas fuentes de información pública permitieron la identificación de las buenas prácticas en la gestión del servicio a cruceros en Cozumel. Sin embargo, hay que señalar que existen escasas publicaciones científicas que evalúen el comportamiento del turismo de cruceros en Las Bahamas, por lo cual para la identificación de las buenas prácticas se revisaron sitios webs sobre cruceros, así como guías de viajes turísticas para la evaluación de la oferta y servicios.

\section{Fase 2: Fase de análisis}

\section{Principales problemas en la gestión del servicio a cruceros en La Habana.}

La Habana, como destino turístico presenta una serie de problemas en la gestión del servicio a cruceros. Entre los principales se encuentra la mala gestión de la infraestructura portuaria. Primeramente, solo cuenta con una terminal, "La Terminal Sierra Maestra", la cual no está preparada para recibir cruceros de gran calado ni grandes volúmenes de embarcaciones.

En segundo lugar, las malas condiciones técnicas y estructurales del puerto, con capacidad para 3 ómnibus de Transtur solamente por cada extremo; lo que trae consigo demoras y molestias al cliente.

Otro elemento responde a que no se garantiza un correcto y ágil flujo de clientes en las diferentes áreas de la terminal, se crean largas filas para los procedimientos de aduana, escáneres, cambio de moneda lo cual afecta el tiempo de salida de las excursiones. Además, las malas condiciones de la terminal, principalmente de la salida sur, las cuales atentan contra una buena ejecución de los servicios, tanto transfer como excursiones y contra la seguridad de los cruceristas.

Influye, además, la congestión de los atractivos y sitios turísticos, sobre todo cuando hay más de un barco en el puerto. Lugares reconocidos como El Floridita, La Bodeguita del medio, Tropicana, La Cabaña, entre otros, que constituyen una visita obligada para un crucerista que compre una excursión en La Habana, sobrepasan su capacidad de carga y no logran brindar el servicio esperado por el cliente. Esto indica además que no existe una oferta extrahotelera completamente desarrollada para satisfacer la demanda de este segmento de mercado. 
Es necesario resaltar que, las agencias de viajes no cuentan con un perfil del crucerista, ni con estudios de la competencia. Si bien es verdad que poseen años de experiencia en el sector y una fuerza laboral preparada, un estudio de mercado siempre resulta necesario para la creación de nuevas ofertas y productos.

La venta de excursiones a los cruceristas no se realiza directamente, sino a través de la compañía armadora, quien realiza la promoción y venta de las mismas a los clientes. Para esto, las agencias en La Habana llenan una ficha que envían a la compañía con todas las especificaciones, es decir, las excursiones con su descripción, precios, si presentan dificultades para ciertos tipos de clientes o edades, y luego la compañía elige cuales venderá. La agencia debe dar un precio neto al crucero, para que esta añada su comisión, de esta manera, la compañía vende a un precio más alto de lo que se oferta en tierra, provocando que muchos clientes desistan de comprar las excursiones y visiten los atractivos del destino por sí solos o en compañía de proveedores de servicios privados. Además, los sitios webs de agencias como Cubatur y Cubanacán no permiten la venta ni reserva de servicios a cruceristas, solo su promoción. De igual manera, la venta de excursiones en el puerto está prohibida para las agencias, como parte del contrato con las compañías armadoras.

La alta racha de cruceros estos últimos cuatro años trajo consigo la apertura de nuevos negocios y con estos, nuevos empleos. Tanto en el sector estatal como en el privado, destacando este último. Se habilitaron nuevos puestos de trabajo en las agencias de viajes, en centros de ocio y de restauración, entre otras entidades pertenecientes al sector turístico, sin embargo, las últimas medidas que afectaron la entrada de cruceros influyeron directamente en este aspecto, tanto en la pérdida y reorientación de puestos de trabajo, como en el cierre y la disminución de ingresos de algunos negocios.

A pesar de contar con un alto índice de satisfacción por parte de los cruceristas, como se explicó anteriormente, aún existen problemas en la prestación del servicio. En encuestas realizadas por la agencia de viajes Cubanacán en el 2018 a los cruceristas, fueron evaluados de regular, mal y pésimo la variedad de la oferta, el servicio en el puerto, el servicio de restauración y la relación calidad-precio.

Existen además otras deficiencias en el destino, como el mal estado de algunas calles, la higiene y el deterioro de algunas fachadas, que constituyen aspectos que transmiten una mala imagen de la ciudad.

Por último, se encuentra el escaso desarrollo tecnológico, con relación a la conexión a Internet, el uso de las redes sociales como medio de distribución y promoción, así como aplicaciones y equipos interactivos de promoción e información para turistas y excursionistas. Por lo cual no se corresponde con la tendencia dada por CLIA (2019) que se refiere a este aspecto. No obstante, hay que reconocer el esfuerzo del país y el interés gubernamental en la informatización de la sociedad con la introducción y el desarrollo de la 3G y la 4G para el acceso a Internet, vía datos móviles, así como la incorporación de nuevas zona Wifi y salas de navegación. 


\section{Identificación de las buenas prácticas en la gestión del servicio a cruceros.}

\section{Cozumel.}

La isla de Cozumel se localiza en el estado de Quintana Roo, situado al Oriente de la península de Yucatán, en México. Su ubicación geográfica le ha permitido erigirse como uno de los destinos principales para cruceros marítimos en el ámbito turístico internacional y el más importante a nivel nacional, teniendo en cuenta también su proximidad a los Estados Unidos, principal mercado emisor de cruceristas a nivel mundial, así como la integración a rutas que incluyen puertos no solo del Caribe, sino también del Océano Pacífico, gracias a lugares estratégicos como el Canal de Panamá.

La misma la integran tres terminales internacionales de cruceros, la marina turística Banco Playa, la terminal de transbordadores y la terminal marítima de San Miguel que brinda servicio a tenders, embarcaciones turísticas y embarcaciones de pasajeros, todas bajo la Administración Portuaria Integral local. Esta isla se reconoce como un lugar de clase mundial por cumplir las exigencias internacionales (APIQROO, 2013). En este sentido, es importante señalar, la especialización de sus instalaciones, construidas específicamente para recibir cruceros turísticos de grandes dimensiones, sin la complicación de compartir el mismo puerto con otro tipo de navío.

Este aspecto lleva a reflexionar al respecto de que contar con la infraestructura adecuada para poder interactuar y competir con las regiones vecinas de alto nivel económico, puede generar una dinámica capaz de favorecer que parte de la riqueza de estas llegue al destino.

Existe una clara especialización económica centrada en las actividades turísticas. Además, su condición de isla favorece su vocación turística, dado que esta actividad se desarrolla a cabalidad, sin necesidad de compartir ese protagonismo con otros sectores relevantes (Padilla y García, 2018).

A nivel federal y enmarcado en el Acuerdo Nacional por el Turismo (ANT) las Secretarías de Turismo, Educación Pública y Trabajo y Previsión Social crearon el Sistema Nacional de Capacitación para el Sector Turismo (SNCT) para fomentar la profesionalización del sector, impulsando la capacitación de los trabajadores, teniendo el año 2018 como límite para el cumplimiento de los objetivos establecidos en el ANT.

La Isla de las Golondrinas, como se le conoce a Cozumel, cuenta con una vasta infraestructura turística, que se ha transformado armoniosamente a la par de sus comodidades y atractivos, al día y a la vanguardia en servicio y calidad.

Al sur de la población se ubican espectaculares clubes de playa que ofrecen a los amantes del sol y la arena magníficos bares y restaurantes, pabellones para masajes en la playa, música y diversión, en donde pueden además practicarse toda clase de deportes acuáticos como buceo, snorkel, kiteboarding, windsurfing (tablavela), paracaidismo acuático, esquí, pesca y mucho más. 
Existen spas e instalaciones de alto estándar, así como una vasta oferta extrahotelera. Dentro de esta, los cruceristas disfrutan de los teatros (tanto al aire libre como cubiertos), cines, plazas de toros, centros nocturnos. Se destaca también el Cozumel Country Club, que cuenta con un campo de Golf de 18 hoyos con Par 72, rodeado de una exuberante y exótica vegetación que anida aves y reptiles únicas en su especie. Igualmente, es importante mencionar que el mismo cuenta con una certificación Audubon International Signature Sanctuary.

A pesar de que el destino es catalogado como destino de Sol y Playa, Cozumel ofrece una gran diversidad de atractivos y actividades de diversas índoles. Es clasificado como uno de los lugares más populares en el mundo del buceo y de las actividades acuáticas. Forma parte del sistema de arrecifes de coral más grande de América. Sus bellezas naturales, la hospitalidad de su gente y la seguridad del destino han hecho de Cozumel uno de los destinos favoritos de los turistas en la Riviera Maya. Es un destino que cuenta con atractivos turísticos que incluyen patrimonio cultural y natural; entre los más populares se encuentran: El Parque Botánico Chankanaab, La Reserva Ecológica Punta Sur, el sitio arqueológico de San Gervasio, y entre los monumentos históricos más representativos el Reloj Público, la explanada de la Bandera, el Museo de la Isla que con sus 4 salas temáticas sintetiza armónicamente la geografía, vida, historia y cultura de la isla, el Monumento al Mestizaje, la Iglesia de San Miguel Arcángel, la Fuente del Buzo, la Plaza de las Dos Culturas y el Parque Benito Juárez.

También cuenta con una gran diversidad en cocina internacional, así como con una fuerte tradición folklórica donde destacan sus fiestas y su carnaval. Para los amantes de la naturaleza, existe la posibilidad de realizar actividades de kayac, trekking y observación de aves y mamíferos endémicos en las zonas de las lagunas ubicadas al norte de la isla. El destino cuenta con un centro de convenciones donde se pueden realizar congresos, simposios y ferias.

De igual forma, las compras en Cozumel, constituyen un gran atractivo, ya que se puede encontrar todo tipo de productos, tiendas de diseñadores, joyas tales como, diamantes, esmeraldas y zafiros, artesanías y tiendas de souvenirs que ofrecen artesanías mexicanas. Esto es debido a que las compras en Cozumel son libres de impuestos por lo que constituye un atractivo turístico para los visitantes y locales.

Lo anteriormente planteado demuestra que Cozumel tiene una oferta diversificada que hace que los cruceristas que llegan al destino puedan elegir de acuerdo a sus deseos y necesidades. Esta diversificación de la oferta viene acompañada de una amplia cartera de producto y servicios, disponibles para el crucerista tanto on-line, como a través de la compañía de cruceros, turoperador u otro medio. De acuerdo a un estudio realizado por la BREA en el 2018 para la FCCA, la compra de excursiones en el destino, el principal canal de distribución es la compañía de cruceros con un $74 \%$.

Es importante señalar que, Cozumel cuenta con un perfil del crucerista bien definido (Anaya y Palafox, 2015). Existen también numerosas agencias de viajes que prestan sus servicios a las 
compañías de cruceros y cruceristas, entre las que se encuentran: Caribbean Tours, Cozumel online, Cozumel Travel, Fantasy Travel Experts, Fiesta Cozumel, Intermar Cozumel, entre otras. Muchas de estas agencias son especializadas, brindando de esta manera un servicio más acorde a las necesidades del mercado que son cada vez más fragmentadas.

Cozumel también se destaca por presentar una alta calidad en sus servicios. Como se mencionó anteriormente, los cruceristas presentan un $97 \%$ de satisfacción.

Con respecto al acceso tecnológico, la isla cuenta con acceso al mundo digital, aspecto que forma parte de la Estrategia de Conectividad Estatal de Quintana Roo, disminuyendo cada vez más la brecha existente entre las zonas urbanas y las rurales. Además como parte del proyecto "Cozumel, destino turístico inteligente", se han desarrollado acciones como la construcción de quioscos interactivos para los visitantes que llegan a la isla a buscar información, videos grabados con tecnología 4K para promocionar Cozumel como isla inteligente, se desarrolló la aplicación móvil Cozumel Smart Island disponible para computadora, tableta y celular (Android e iOS) que permite hacer consultas de trámites y servicios, reportes, entre muchos otros servicios. A lo cual se le suma un catálogo en el que se busca integrar el grueso de productos y servicios turísticos de la isla (Narváez, 2017).

\section{Las Bahamas.}

Las Bahamas es un país constituido por más de 700 islas (de las cuales, 24 están habitadas y más de 600 deshabitadas), cayos e islotes en el Océano Atlántico; al norte de Cuba y República Dominicana, noroeste de Islas Turcas y Caicos, al sureste del estado estadounidense de Florida y al este de los Cayos de la Florida.

Este destino posee una desarrollada infraestructura portuaria. El puerto principal se encuentra en Freeport, Matthew Town y Nassau; estos son: Freeport, South Riding Point, Ocean Gay, Nassau y Clifton. De estos, Freeport se encuentra entre los más importante del Caribe, con un alto Índice de Desempeño Logístico, además es el único en el destino con 16 metros de calado preparado para los buques de gran calado, Unidad de Estudios de Políticas Económicas y Sociales del Caribe (UEPESC, 2014).

Por otro lado, en el puerto de Nassau pueden atracar una docena de cruceros simultáneamente, por lo que la ciudad suele estar siempre llena de cruceristas; el mismo posee una ubicación favorable al estar justo al lado del centro. Con relación a este puerto existe un nuevo proyecto con Global Port Holdings Limited, valorado en 250 millones de dólares, donde se añadirán dos nuevos muelles de atraque con capacidad para dos nuevos barcos de gran tamaño, un auditorio para conciertos con capacidad para 10.000 espectadores, un cine al aire libre y un complejo de nuevos edificios, así como habilitar servicio de tranvías para el transporte de los cruceristas (García, 2018).

Dentro de las categorías de compra más populares en el destino, se destacan con más de un $45 \%$ cada una de ellas: las excursiones, los alimentos y bebidas y souvenirs. El turismo de cruceros 
reporta una contribución económica directa de \$ 405,75 millones de dólares al destino, la segunda más alta del Caribe después de Cozumel (BREA, 2019).

Con respecto a la infraestructura turística, el destino posee más de 60 aeropuertos distribuidos en las islas, más de $2000 \mathrm{~km}$ en carreteras, además existe un puente que une a Nassau con Paradise Island, permitiendo la comunicación entre estas dos islas. Tiene una oferta hotelera de importantes resorts y parques acuáticos (UEPESC, 2014), donde destaca el Atlantis Paradise Bahamas, un impresionante hotel de lujo con acuario y jardines, restaurantes, delfinario, playas con todos los deportes acuáticos, y un parque acuático (Rodríguez, 2019). Además de una gran variedad en museos, bares, casinos, restaurantes, discotecas y lugares de esparcimiento al aire libre.

En las Bahamas el crucerista tiene múltiples opciones de actividades. Infinitas oportunidades para los amantes de los deportes acuáticos, especialmente los submarinistas, windsurf y pesca deportiva. Así como para los amantes de la naturaleza, Bahamas posee atracciones naturales como: la tercera barrera de coral más grande del mundo del tipo franja; en la isla Andros, los hoyos azules, en una gran cantidad de cuevas marinas para buceo y una gran reserva de diferentes especies de aves, en Paradise Island (Rodríguez, 2019).

En la capital, Nassau, se venden gran número de excursiones relacionadas con la historia del lugar y visitas a fortalezas que datan del siglo XVII. El destino se encuentra posicionado también como uno de los mejores destinos de luna de miel. Además, se brinda asistencia para la celebración de reuniones, eventos y congresos.

Las compras constituyen uno de los principales atractivos en Nassau, ya que están libres de impuestos. Se encuentran gran cantidad de tiendas de todo tipo de marcas y servicios. Se destacan en esta actividad el Straw Market, así como Bay Street, la calle de Nassau por excelencia para realizar compras (Rodríguez, 2019).

En Tripadvisor se encuentran también una gran cantidad de actividades, más de 500 opciones al aire libre, más de 360 recorridos en barcos y deportes acuáticos, más de 213 en naturaleza y parques, así como otras opciones de compras, spas y bienestar, zoológicos y acuarios, clases y talleres, conciertos y espectáculos, museos y casinos. Algunas de ellas muy originales como el nado con cerditos en la Isla de los cerdos y el nado con tortugas. Todo lo anterior demuestra que, en estas islas, existe una amplia oferta turística de servicios con una gran variedad, para todos los gustos y segmentos del mercado.

Como se mencionó anteriormente, el por ciento de satisfacción de los cruceristas que visitan Las Bahamas es del 89\%, sin embargo, de acuerdo al sitio de reseñas de cruceros Cruise Critic, Las Bahamas tienen una valoración de los cruceristas de excelente. De más de 17000 opiniones el $61 \%$ aproximadamente la valoran de excelente y muy bien. 
Determinación de la brecha de desempeño en la gestión del servicio a cruceros de La Habana con los destinos líderes del Caribe

La tabla 3 ilustra la brecha de desempeño en la gestión del servicio a cruceros de La Habana con respecto a los destinos seleccionados, a partir de la evaluación realizada por los expertos.

Tabla 3: Determinación de la brecha de desempeño en la gestión del servicio a cruceros del destino La Habana

\begin{tabular}{lcc}
\hline \multicolumn{1}{c}{ Variables } & $\begin{array}{c}\text { Brecha entre } \\
\text { La Habana - } \\
\text { Cozumel }\end{array}$ & $\begin{array}{c}\text { Brecha entre } \\
\text { La Habana - Las } \\
\text { Bahamas }\end{array}$ \\
\hline $\begin{array}{l}\text { Arribo de cruceristas al destino } \\
\text { Gasto promedio del crucerista en el } \\
\text { destino }\end{array}$ & 1 & 1 \\
$\begin{array}{l}\text { Generación de empleo } \\
\text { Contribución económica al destino }\end{array}$ & 1 & 1 \\
Infraestructura portuaria & 1 & 1 \\
Cantidad de servicios turísticos & 1 & 1 \\
Calidad de servicios turísticos & 2 & 1 \\
Rol de las agencias de viajes & 1 & 1 \\
Acceso a la tecnología & 1 & 3 \\
Brecha promedio & $\mathbf{1 . 1}$ & - \\
\hline
\end{tabular}

Fuente: Elaboración propia.

Como se aprecia en la tabla anterior, con excepción a la calidad de los servicios donde La Habana posee una brecha con operaciones en paridad con Cozumel y una brecha positiva con Las Bahamas, las demás variables poseen una brecha negativa.

Estos destinos constituyen líderes no solo por las cifras significativas en relación con la llegada de cruceristas, el gasto promedio de los mismos en el destino o la contribución económica que reporta la actividad, sino también por aspectos como la desarrollada infraestructura portuaria y turística, la amplia y diversificada oferta turística de servicios, la alta generación de empleo, así como el número de agencias que prestan servicios a cruceros en el destino.

Luego, se pudo apreciar que La Habana, con relación a Cozumel y Las Bahamas, en la gestión del servicio a cruceros, posee una brecha negativa, esto quiere decir que las prácticas de los líderes son mejores que las del destino.

Se determinaron como principales brechas la no accesibilidad al mercado norteamericano, la deteriorada infraestructura portuaria en el destino, la escasa oferta extrahotelera, así como la brecha tecnológica existente. 


\section{Fase 3: Fase de integración}

Con el propósito de mejorar la gestión del servicio a cruceros, así como aumentar la competitividad del destino La Habana dentro del área del Caribe en esta actividad, y teniendo en cuenta una visión estratégica, se proponen a continuación las siguientes acciones:

- Elaborar el perfil del crucerista que llega al puerto de La Habana, por parte de las principales agencias que atienden la actividad de cruceros y del MINTUR.

- Realizar estudios de la competencia y del mercado en general en la actividad de cruceros por parte del MINTUR.

- Definir estrategias de promoción y publicidad para posicionar al destino como uno de los principales puertos del Caribe en cuanto al turismo de cruceros.

- Captar, como clientes, nuevas compañías navieras provenientes de Europa y Asia.

- Diversificar la oferta turística del destino ampliando las posibilidades de elección para el crucerista.

- Desarrollar el turismo de compra aprovechando los productos típicos de Cuba.

- Diseñar una nueva cartera de excursiones teniendo en cuenta las características y necesidades de los cruceristas.

- Crear excursiones especializadas para clientes con problemas motores, solteros, familias, personas de la 3ra edad, clientes LGBT.

- Garantizar una mayor organización en los itinerarios de las excursiones para disminuir la congestión de los atractivos y lugares turísticos.

- Impartir conferencias a los prestatarios de servicios sobre las principales tendencias dentro de la actividad de los cruceros.

- Incorporar a otras agencias del destino, como Ecotur y San Cristóbal, en la asistencia a cruceros.

- Ampliar la oferta extrahotelera con nuevos centros de ocio y de restauración.

- Creación de mercados locales para la venta de artesanías y souvenirs.

- Crear nuevos empleos vinculados, directa e indirectamente, en la gestión del servicio a cruceros.

- Restaurar la Terminal de cruceros Sierra Maestra.

- Construir o habilitar una zona de parqueo en las cercanías de la Terminal Sierra Maestra.

- Garantizar un correcto y ágil flujo de clientes en las diferentes áreas de la terminal.

- Desarrollar la conexión a Internet vía datos móviles 3G y 4G.

- Ampliar el número de zonas Wifi en el destino garantizando las principales zonas turísticas. 
- Habilitar la venta on-line de excursiones a cruceristas.

- Realizar acciones vinculadas a la limpieza del destino y mejora de su infraestructura.

- Establecer una mejor comunicación entre las diferentes entidades que atienden el servicio a cruceros desde las autoridades portuarias hasta los prestatarios de servicios.

\section{Conclusiones.}

- El turismo de cruceros se ha convertido en una de las áreas con mayor proyección en volumen de viajeros e ingresos, destacándose en esta actividad el área del Caribe como el primer destino mundial.

- El desarrollo del turismo de cruceros en La Habana, ha estado sujeto en gran medida a las restricciones del gobierno norteamericano. Las últimas medidas dictadas por este gobierno en junio del 2019, disminuyeron en un $49 \%$ la llegada de cruceristas a La Habana y en un $50 \%$ a Cuba.

- La Habana, con respecto a Cozumel y Las Bahamas, presenta una brecha negativa en la gestión del servicio a cruceros. Las brechas principales son: la no accesibilidad al mercado norteamericano, la deteriorada infraestructura portuaria en el destino, la escasa oferta extrahotelera, así como la brecha tecnológica existente.

- A partir de la identificación de las buenas prácticas en los destinos de Cozumel y Las Bahamas, se propuso un total de 22 acciones para la mejora de la gestión del servicio a cruceros en La Habana, la cuales contribuirán al desarrollo del sector del turismo en el destino Cuba.

\section{Referencias Bibliográficas.}

APIQROO (2013). Totales por tipo de embarcación. Administración Portuaria Integral de Quintana Roo, México. Administración Portuaria Integral de Quintana Roo. Consultado el 14 de enero del 2020. Recuperado de: http://www.apiqroo.com.mx/web/ApiqrooVE/tabla.php?tip=2\&y=2013\&port=1

Business Research and Economic Advisors. (2018). Economic contribution of Cruise Tourism to the destination economies. A Survey-based Analysis of the Impacts of Passenger, Crew and Cruise Line Spending Volume II Destination Reports. Prepared for Florida-Caribbean Cruise Association And Participating Destinations.

Chevalier, S. (junio 2019). Cuba y el turismo de cruceros. Consultado de 25 de enero de 2020. Recuperado de: https://es.statista.com/grafico/18305/mercado-de-los-cruceros-en-cuba-yel-caribe/

CLIA (diciembre 2019). Releases 2020 State of the Cruise Industry Outlook Report. Consultado el 10 de febrero de 2020. Recuperado de: https://cruising.org/en/news-and-research/pressroom/2019/december/clia-releases-2020-state-of-the-cruise-industry-outlook-report 
Developing Natural Activities. (2013). Análisis de benchmarking y best practices, estudio de los competidores y de las buenas prácticas de otros destinos turísticos. Plan Estratégico de Ordenación y Promoción del Turismo en el Valle del Aconcagua. Barcelona.

Excelencias Cuba (agosto 2018). El turismo de cruceros en Cuba y La Habana. Consultado el 12 de enero de 2020. Recuperado de: http://www.excelencias.com/

García, J. (2018). Cruceros en el Caribe Oriental. Estos son los TPO 7 puerto y escalas. Consultado el 20 de febrero de 2020. Recuperado de: https://cruceroadicto.com/cruceros-en-el-caribeoriental.html

Hernández, C. y Cano, M. (2017). La importancia del benchmarking como herramienta para incrementar la calidad en el servicio en las organizaciones. Revista Ciencia Administrativa, 2. Recuperado de: https://www.uv.mx/iiesca/files/2018/03/04CA201702.pdf

Instituto Distrital de Turismo. (2011). Cartilla de buenas prácticas de sostenibilidad para empresarios turísticos. Fortalecimiento de la competitividad turística de la localidad de Teusaquillo. Instituto Distrital de Turismo: Bogotá.

Instituto Tecnológico de Monterrey (2000). Benchmarking del Turismo Ecuatoriano. Instituto Tecnológico de Monterrey: Bogotá.

MINTUR (2019). Entrevista realizada a MSc. Oscar Henríquez Díaz, Especialista Ramal Superior MINTUR. La Habana, Cuba.

Narváez, M. (2017). Cozumel, primea isla inteligente del Caribe. Tecnología ambiental Sostenibilidad empresarial. Enero 2020. Consultado el 06 de febrero de 2020. Recuperado de: http://www.cienciamx.com/index.php/tecnologia/tic/17394-cozumel-primera-islainteligente-caribe

López, L. (2010). Benchmarking y su Aplicación en Turismo. Revista de Estudios Politécnicos, VIII (14): 163-180.

Rodríguez, M (2019). Visitar Nassau- Bahamas - Súper guía para preparar tu visita. Consultado el 12 de enero de 2020. Recuperado de: https://cruceroadicto.com/visitar-nassau-bahamasguia-de-que-ver-y-hacer.html

Ruiz, S. C. y Parada, O. (2017). Principales variables para la gestión de la calidad del servicio de recepción de turismo de cruceros. Cuadernos de Turismo (38), 429 - 457. DOI : http://dx.doi.org/10.6018/turismo.38.271511

Unidad de Estudios de Políticas Económicas y Sociales del Caribe. (2014). Informe País: Bahamas. Instituto de Economía, Planificación y Desarrollo.

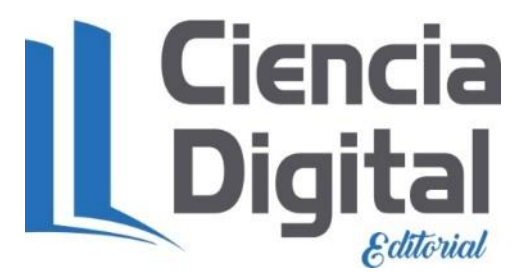




\section{PARA CITAR EL ARTÍCULO INDEXADO.}

Pérez García, C., Hernández Flores, Y., Rodríguez González, M., \& Velasteguí López, L. E. (2020). Buenas prácticas en la gestión del servicio a cruceros de los principales destinos del Caribe. Acciones para su mejora en el destino La Habana. Conciencia Digital, 3(4), 71-89. https://doi.org/10.33262/concienciadigital.v3i4.1426

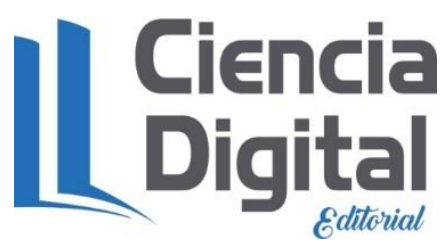

El artículo que se publica es de exclusiva responsabilidad de los autores y no necesariamente reflejan el pensamiento de la Revista Conciencia Digital.

El artículo queda en propiedad de la revista y, por tanto, su publicación parcial y/o total en otro medio tiene que ser autorizado por el director de la Revista Conciencia Digital.
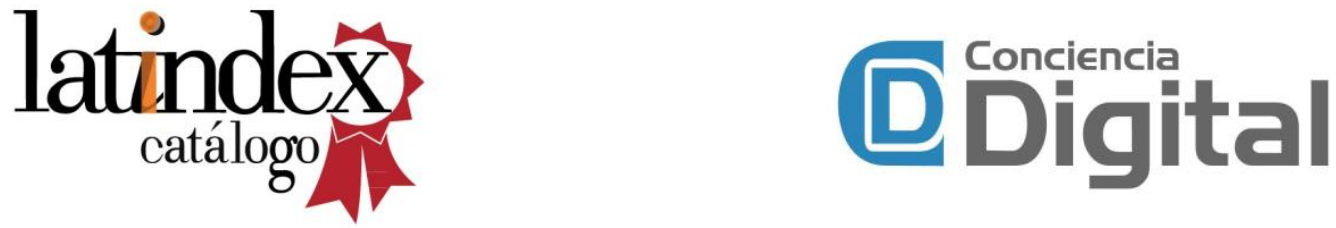